\title{
The current application of thermal ablation in colorectal liver metastasis
}

\author{
Zhongyi Zhang, Kun Yan
}

Key Laboratory of Carcinogenesis and Translational Research (Ministry of Education/Beijing), Department of Ultrasonography, Peking University Cancer Hospital \& Institute, Beijing, China

Correspondence to: Kun Yan, MD. Key Laboratory of Carcinogenesis and Translational Research (Ministry of Education/Beijing), Department of Ultrasonography, Peking University Cancer Hospital \& Institute, Fucheng Road No. 52, Haidian District, Beijing, China. Email: ydbz@vip.sina.com. Comment on: Takahashi H, Berber E. Role of thermal ablation in the management of colorectal liver metastasis. Hepatobiliary Surg Nutr 2020;9:49-58.

Submitted Sep 07, 2021. Accepted for publication Oct 18, 2021.

doi: 10.21037/hbsn-21-365

View this article at: https://dx.doi.org/10.21037/hbsn-21-365

Colorectal liver metastases (CRLM) are one of the most frequent clinical conditions. Over the last 20 years, the clinical outcome for patients with CRLM has improved greatly. Liver resection is the gold standard treatment for resectable CRLM, achieving a 5-year overall survival (OS) rate of $42 \%$ (1). However, the majority of patients with CRLM are not candidates for resection. With the development of thermal ablation, this technique provides an alternative treatment modality by either ablation alone or combined with resection. A recent review by Takahashi et al. summarized the current role of thermal ablation in the management of CRLM (2).

In this review, the authors stated that ablation should not be used in lieu of liver resection for resectable CRLM based on current available evidence. Regarding the rapid advancement of systemic and local therapy, the treatment strategies for patients with CRLM are suggested to be discussed by the multidisciplinary team (MDT). Also, the authors listed the indications of ablation in their center, including unresectable liver lesions (tumor number $\leq 8$, tumor size $\leq 4 \mathrm{~cm}$ ), combined with hepatectomy, and resectable liver lesions but patients unfit to receive resection because of comorbidities and patient preference. These indications are highly feasible and can be referred by readers. It is noteworthy that the preferred tumor size and number were inconsistent in the light of the clinician's experience. In general, thermal ablation is recommended for tumor size $\leq 3 \mathrm{~cm}$. For tumor size between 3 and $5 \mathrm{~cm}$, welllocated tumors can also be effectively treated depending on anatomical position and the treatment protocol used (3). Tumor number varies from 5 to 9 in the literature, which depends on the total liver tumor volume.

The authors then pointed out the pros and cons of 3 approaches to perform ablation. Open and laparoscopy approaches allow better staging for occult liver metastases or peritoneal disease and protect surrounding organs from thermal injuries. It has been reported that additional metastases were detected in $10-25 \%$ of patients with CRLM by intraoperative ultrasound (4), which may result in changing the surgical strategy. The percutaneous approach provides the least invasive approach and has a significant role in patients with limited recurrent liver metastases after resection or ablation. Moreover, percutaneous ablation can be used as the 'test-of-time' approach reported by Livraghi et al. (5). The rationale of this approach is: (I) high technical success rate of ablation for small tumors without the need for resection; (II) if ablation was unsuccessful, the resection was still feasible; (III) if patients develop additional lesions after ablation and would no longer be suitable for resection, they can be spared unnecessary surgery. Among the 88 patients treated in the study by Livraghi et al. (5), 26\% remained free of disease without the need for resection, $24 \%$ underwent resection after ablation, and $50 \%$ were unfit for resection due to disease progression.

Compared with liver resection, local tumor recurrence (LTR) is a major issue after ablation. Predictors of LTR include tumor size $\geq 3 \mathrm{~cm}$ and ablation margin $\leq 0.5 \mathrm{~cm}$. In 
addition to the increased tumor burden in patients with a tumor size $\geq 3 \mathrm{~cm}$, overlapping ablations are usually used. This makes it more difficult to ensure adequate ablation margins in all directions, which might contribute to LTR. To reduce the likelihood of LTR, a minimum $1 \mathrm{~cm}$ ablation margin is recommended in all directions (3). Shady et al. (6) reported that no LTP was observed for CRLM tumors ablated with a margin $>1 \mathrm{~cm}$, while the LTP rate for tumors with a margin $0.5-1 \mathrm{~cm}$ was significantly lower than for those with a margin $\leq 0.5 \mathrm{~cm}(14.8 \%$ vs. $71 \%, \mathrm{P}<0.001)$.

Until now, there have been few available randomized controlled trials (RCTs) on ablation versus other therapy. For unresectable CRLM, the EORTC-CLOCC trial revealed that ablation combined with systemic chemotherapy could improve OS and progression-free survival (PFS) compared to chemotherapy alone (7). However, for resectable CRLM, there is still a lack of evidence from RCTs comparing ablation with liver resection. Recently in 2020, the LAVA trial (ISRCTN52040363) (8), which compared liver resection with thermal ablation in high surgical risk patients with CRLM, closed early because only 9 participants were randomized over 1 year. There are many reasons that inhibit recruitment, including fewer participants eligible for both ablation and resection, surgeon's preference with unconscious bias towards surgery, and misconceptions about the eligibility criteria. The other 2 RCTs COLLISION (NCT03088150) and HELARC (NCT02886104) focusing on hepatectomy versus ablation of CRLM are still ongoing and we are looking forward to the outcomes of those trials.

As a local treatment, thermal ablation has been confirmed to be an effective and safe modality for controlling CRLM, either used alone or combined with chemotherapy and/ or surgery. It is worth mentioning that local treatment including ablation and liver resection is only part of the entire management of CRLM in the era of modern medicine. Moreover, the clinical application of thermal ablation differs from each center, including approaches (open vs. laparoscopy vs. percutaneous), techniques (radiofrequency ablation $v s$. microwave ablation), ablation devices, and performers (surgeon $v$ s. radiologist) and their experiences. Therefore, it is recommended that decisions be made for patients with CRLM through MDT discussion. In the future, further well-designed studies on thermal ablation in selected patients with CRLM are needed to expand its application.

\section{Acknowledgments}

Funding: This work was supported by Capital's Funds for Health Improvement and Research (2020-2-2152).

\section{Footnote}

Provenance and Peer Review: This article was commissioned by the editorial office, Hepatobiliary Surgery and Nutrition. The article did not undergo external peer review.

Conflicts of Interest: Both authors have completed the ICMJE uniform disclosure form (available at https://hbsn. amegroups.com/article/view/10.21037/hbsn-21-365/coif). The authors have no conflicts of interest to declare.

Ethical Statement: The authors are accountable for all aspects of the work in ensuring that questions related to the accuracy or integrity of any part of the work are appropriately investigated and resolved.

Open Access Statement: This is an Open Access article distributed in accordance with the Creative Commons Attribution-NonCommercial-NoDerivs 4.0 International License (CC BY-NC-ND 4.0), which permits the noncommercial replication and distribution of the article with the strict proviso that no changes or edits are made and the original work is properly cited (including links to both the formal publication through the relevant DOI and the license). See: https://creativecommons.org/licenses/by-nc-nd/4.0/.

\section{References}

1. Adam R, Kitano Y. Multidisciplinary approach of liver metastases from colorectal cancer. Ann Gastroenterol Surg 2019;3:50-6.

2. Takahashi H, Berber E. Role of thermal ablation in the management of colorectal liver metastasis. Hepatobiliary Surg Nutr 2020;9:49-58.

3. Gillams A, Goldberg N, Ahmed M, et al. Thermal ablation of colorectal liver metastases: a position paper by an international panel of ablation experts, The Interventional Oncology Sans Frontières meeting 2013. Eur Radiol 2015;25:3438-54.

4. Ferrero A, Langella S, Giuliante F, et al. Intraoperative liver ultrasound still affects surgical strategy for patients 
with colorectal metastases in the modern era. World J Surg 2013;37:2655-63.

5. Livraghi T, Solbiati L, Meloni F, et al. Percutaneous radiofrequency ablation of liver metastases in potential candidates for resection: the "test-of-time approach". Cancer 2003;97:3027-35.

6. Shady W, Petre EN, Do KG, et al. Percutaneous Microwave versus Radiofrequency Ablation of Colorectal Liver Metastases: Ablation with Clear Margins (A0) Provides the Best Local Tumor Control. J Vasc Interv

Cite this article as: Zhang Z, Yan K. The current application of thermal ablation in colorectal liver metastasis. HepatoBiliary Surg Nutr 2021;10(6):872-874. doi: 10.21037/hbsn-21-365
Radiol 2018;29:268-275.e1.

7. Ruers T, Van Coevorden F, Punt CJ, et al. Local Treatment of Unresectable Colorectal Liver Metastases: Results of a Randomized Phase II Trial. J Natl Cancer Inst 2017;109:djx015.

8. Davidson B, Gurusamy K, Corrigan N, et al. Liver resection surgery compared with thermal ablation in high surgical risk patients with colorectal liver metastases: the LAVA international RCT. Health Technol Assess 2020;24:1-38. 